\title{
A Rotating Flux Pump Employing a Magnetic Circuit and a Stabilized Coated Conductor HTS Stator
}

\author{
Z. Jiang ${ }^{1}$, C. W. Bumby ${ }^{1}$, R. A. Badcock ${ }^{1}$, N. J. Long ${ }^{1}$, H. J. Sung ${ }^{2}$, and M. Park ${ }^{2}$ \\ ${ }^{1}$ Robinson Research Institute, Victoria University of Wellington, Lower Hutt 5046, New Zealand \\ ${ }^{2}$ Department of Electrical Engineering, Changwon National University, Changwon 641-773, Korea
}

(Received 1 March 2016, Received in final form 24 May 2016, Accepted 8 June 2016)

\begin{abstract}
High temperature superconductor (HTS) magnet systems usually employ metal current leads which bridge between the cryogenic environment and room temperature. Such current leads are the dominant heat load for these magnet systems due to a combination of electrical resistance and heat conduction. HTS flux pumps enable large currents to be injected into a HTS magnet circuit without this heat load. We present results from an axialtype HTS mechanically rotating flux pump which employs a ferromagnetic circuit and a Cu-stabilized coated conductor (CC) HTS stator. We show the device can be described by a simple circuit model which was previously used to describe barrel-type flux pumps, where the model comprises an internal resistance due to dynamic resistance and a DC voltage source. Unlike previously reported devices, we show the internal resistance and DC voltage in the flux pump are not exactly proportional to frequency, and we ascribe this to the presence of eddy currents. We also show that this axial-type flux pump has superior current injection capability over barrel-type flux pumps which do not incorporate a magnetic circuit.
\end{abstract}

Keywords : HTS flux pump, coated conductors, dynamic resistance

\section{Introduction}

Superconducting flux pumps enable large currents to be injected into a superconducting magnet system without the requirement for copper current leads which bridge between the cryogenic environment and room temperature [1-8]. Previous works have already demonstrated mechanically rotating barrel-type flux pumps which employ a CC HTS stator and a rotor comprising arrays of rotating permanent magnets, and these flux pumps have been used to inject currents into HTS coils [3-8]. In practical applications of a rotating flux pump, a preferred arrangement would allow the rotor to be located at room temperature, whilst the HTS stator is connected to an HTS coil that is located within the cryogenic environment. We have found a barrel-type rotating flux pump ceased to inject current to a HTS coil at a flux gap (between the rotor magnets and coated conductor stator) larger than $7.5 \mathrm{~mm} \mathrm{[6].} \mathrm{We}$ have shown that we can describe the operation of flux pumps using a simple electrical circuit model shown in

(C)The Korean Magnetics Society. All rights reserved.

*Corresponding author: Tel: +64-4-463-0077

Fax: +64-4-463-9028, e-mail: zhenan.jiang@vuw.ac.nz
Fig. 1(a) in which the flux pump is considered to be a DC voltage source $V_{\mathrm{oc}}$ with an effective internal resistance, $R_{\mathrm{d}}$ which is due to Dynamic resistance $[4,6,9,10]$. Both $V_{\mathrm{oc}}$ and $R_{\mathrm{d}}$ are proportional to magnet-crossing frequency in the flux pumps [4, 6-8]. In a previous work, Hoffmann et al. pointed out the usage of a stabilized CC stator in their barrel-type rotating flux pump causes non-linear $V_{\text {oc }}-f$ relationship due to eddy currents in the stabilizer layers in the CC. In practice, stabilized wires are much preferred due to its resilience to repeated soldering and wire $I_{\mathrm{c}}$ deterioration.

In this work, we built an axial-type flux pump with ferromagnetic circuit using a Cu-stabilized CC HTS stator to investigate the flux gap and frequency dependence of $V_{\mathrm{oc}}$ and $R_{\mathrm{d}}$. The flux gap was varied from $4 \mathrm{~mm}$ to 10.9 $\mathrm{mm}$. Frequency was set at 24, 48, and $96 \mathrm{~Hz}$. We present the DC behavior of a closed superconducting circuit comprising the flux pump connected in series with an HTS coil.

\subsection{Experimental method}

Figures 1(b) and (c) show schematics of our experimental device, which utilize ferromagnetic iron yokes within the rotor and stator to form a magnetic circuit 
(a)

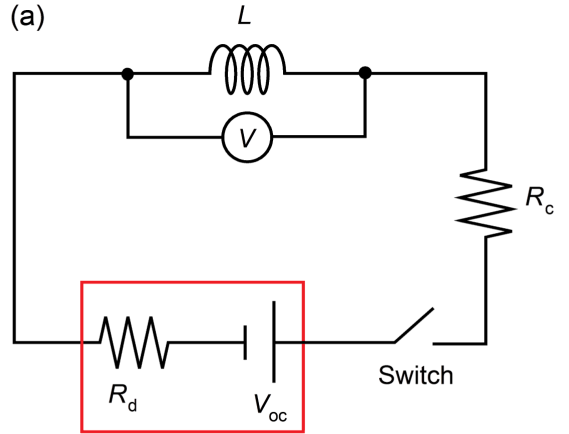

Flux pump
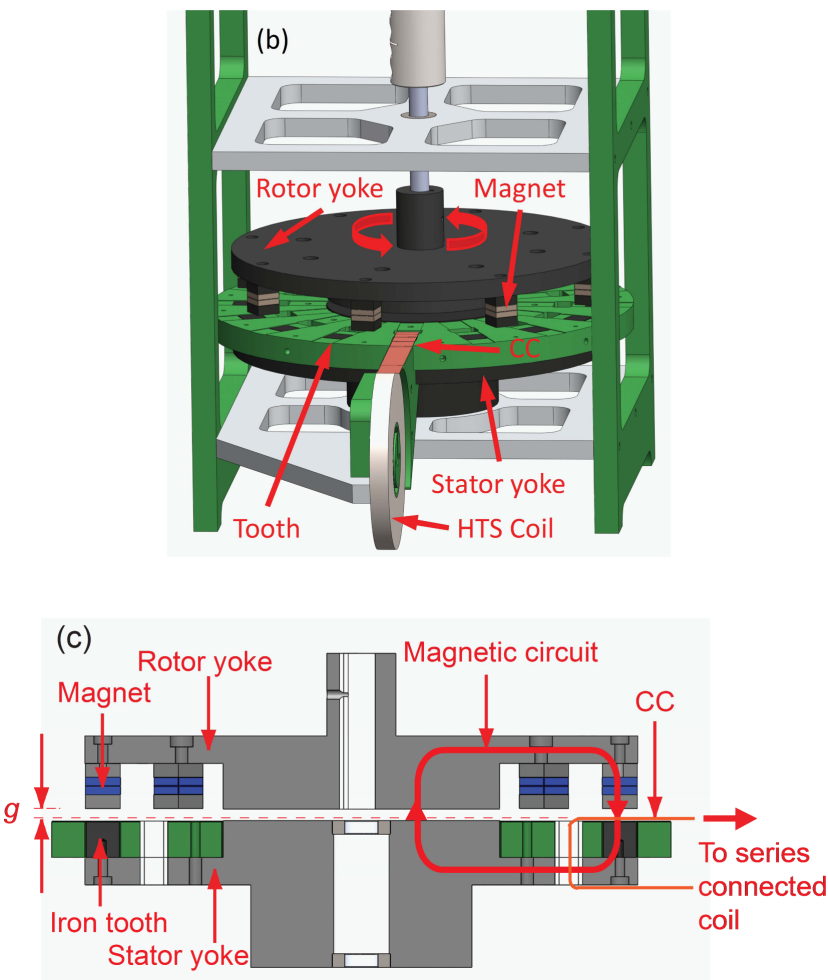

Fig. 1. (Color online) (a) Circuit diagram of a HTS flux pump; (b) 3D view of the axial-type HTS flux pump; (c) cross-section diagram of the flux pump.

which focusses magnetic flux upon the HTS wire. This approach leads to a substantial increase in the applied perpendicular field at the stator wire, when compared to an isolated permanent magnet. The flux pump comprises an array of 8 two vertical stacks of $\mathrm{NdFeB}$ permanent magnets $\left(\mathrm{N} 42,0.5^{\prime \prime} \times 0.5^{\prime \prime} \times 0.125^{\prime \prime}\right)$ equally distributed along the outer edge of the rotor yoke. The stator yoke has a set of protruding iron 'teeth' and a single stabilized HTS CC stator wire was located upon one of these teeth. A hole within the yoke allows the HTS CC stator wire to exit the high field region without being exposed to a magnetic field from the return path of the magnetic circuit.
The iron 'teeth' on the yoke focus the applied magnetic flux from the rotor magnets. This maximizes the local applied magnetic field as the rotor magnet passes over the 'tooth'. The stabilized CC is a commercial SuperPower CC (SCS 12050) with a $20 \mu \mathrm{m}$ copper stabilization layer on both sides, and has a self-field $I_{\mathrm{c} 0}$ of $260 \mathrm{~A}$, and was connected via soldered joints to the HTS coil, which has a measured self-inductance $L=2.4 \mathrm{mH}$ at $77 \mathrm{~K}$. A cryogenic Hall sensor was fixed at the centre of the HTS coil and used to measure the current within the superconducting circuit. The axial flux gap between the rotor magnets and the CC stator (denoted $g$, in Fig. 1(c)) was varied from 4 $\mathrm{mm}-10.9 \mathrm{~mm}$ through the use of a series of interchangeable fixed spacer plates. The rotor was driven by a servo motor via a shaft which extended to the room temperature environment. Both the rotor and stator were placed in a liquid nitrogen $\left(\mathrm{LN}_{2}\right)$ bath for the measurements. The speed of the servo motor was controlled such that the passing frequency of the rotor magnets, $f$, could be adjusted between zero and $96 \mathrm{~Hz}$.

\section{Experimental Result}

Figure 2(a) shows a comparison of the injected coil currents obtained when operating the flux pump at $96 \mathrm{~Hz}$ (720 rpm) using various flux gaps. The injected currents decrease with increasing the flux gap. 13.6 A was injected to the coil at $g=10.9 \mathrm{~mm}$ after 23 minutes. This is a remarkable improvement in injection capability from a barrel flux pump without magnetic circuit which could not inject current at flux gaps larger than $7.5 \mathrm{~mm}$ [6]. This is because of the effectiveness of the magnetic circuit which enables substantial increase in the applied perpendicular field at the stator wire, resulting in the generation of a DC voltage at a larger flux gap [6,7].

Figure 2(b) shows the corresponding output voltages measured across the coil during the same series of experiments. The output voltage at each flux gap exhibits the expected exponential decay described by Eq. (3) in [6]. The time constant $\tau=L / R$, where $R$ is the sum of $R_{\mathrm{d}}$ and the soldered joint resistance, $R_{\mathrm{c}}$ in Fig. 1(a). This can be obtained from the gradient of the lines in Fig. 2(b). We observe that $\tau$ increases as the flux gap increases, which is a result of the reduction in $R_{\mathrm{d}}$ as the peak applied field at the stator decreases. Similarly, the voltage at zero time, i.e., $V_{\text {oc }}$, decreases with increasing flux gap, again reflecting the reduced applied flux which passes through the stator as $g$ is increased.

Figure 2(c) shows the same experimental data as given in Figs. 2(a) and 2(b), but now plotted in terms of the output current versus the output voltage of the coil operat- 

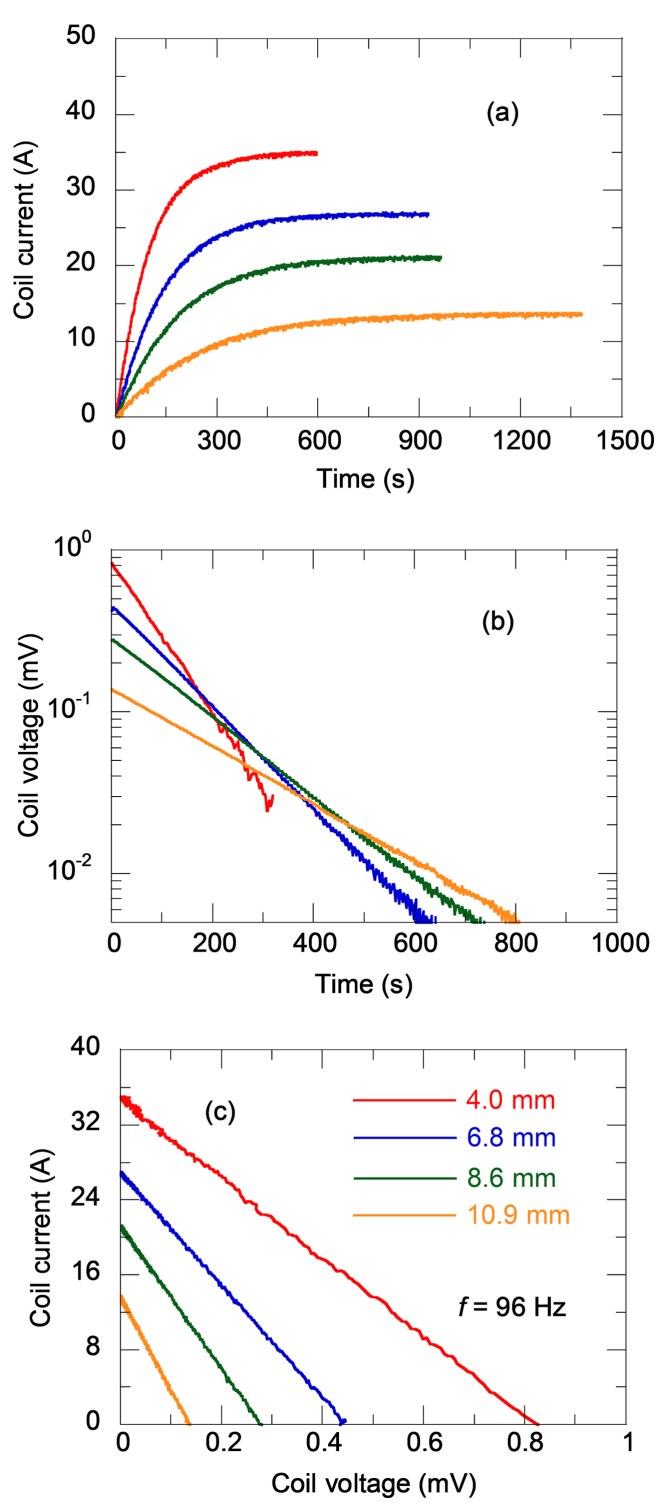

Fig. 2. (Color online) (a) Injected coil currents at $96 \mathrm{~Hz}$; (b) coil voltage at $96 \mathrm{~Hz}$; (c) $I-V$ relationship of the flux pump at $96 \mathrm{~Hz}$.

ing at each flux gap. A straight line relationship between output current and voltage is observed, which indicates that the total resistance of the circuit in Fig. 1(a) is independent of current in each case (as $R=R_{\mathrm{d}}+R_{\mathrm{c}}=$ $-\mathrm{d} V / \mathrm{d} I$ following the sign convention used in this plot). Experimental values for the open-circuit voltage, $V_{\text {oc }}$ and short-circuit current, $I_{\mathrm{sc}}\left(I_{\mathrm{sc}}=V_{\mathrm{oc}} / R_{\mathrm{d}}\right)$ can also be extracted from this plot as these values are derived by the intercepts of each line on the $V$ and $I$ axes respectively [6].

Figure 3(a) shows the frequency dependence of $V_{\text {oc }}$ for various flux gaps. $V_{\text {oc }}$ rises with increasing frequency, but is not linearly dependent on frequency. The non-linear behaviour of $V_{\mathrm{oc}}$ is more apparent when the flux gap is
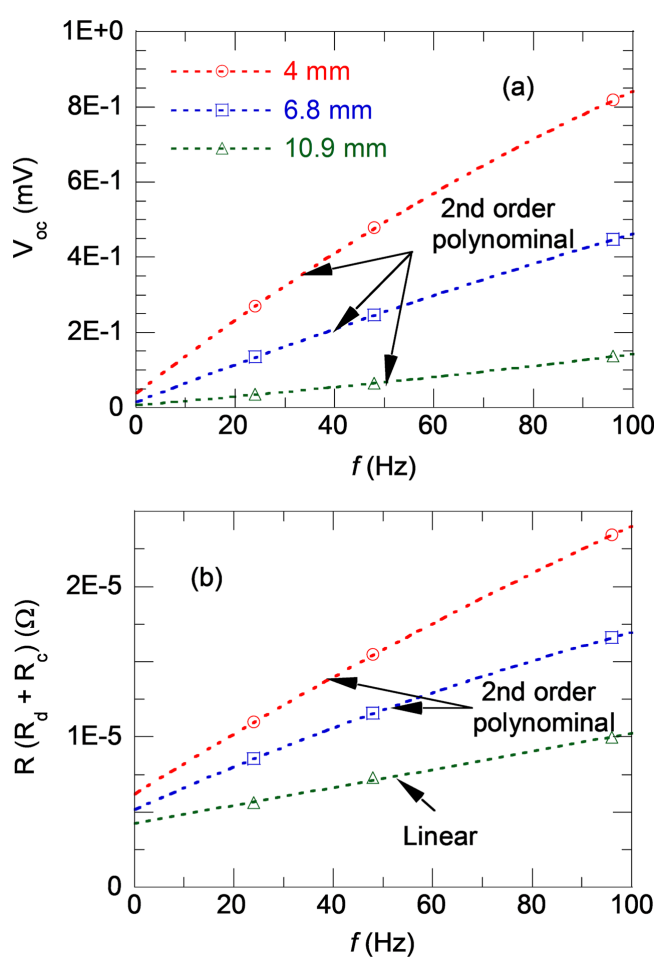

Fig. 3. (Color online) (a) Frequency dependence of $V_{\text {oc }}$; (b) Frequency dependence of $R$.

smaller. This is similar to the result obtained by Hoffmann et al in a stabilized CC [3]. Figure 3(b) shows the frequency dependence of total resistance $R\left(R_{\mathrm{d}}+R_{\mathrm{c}}\right)$ in the circuit obtained from $I-V$ relation shown in Fig. 2(c). Similar to $V_{\mathrm{oc}}, R$ rises with increasing frequency, but is not proportional to $f$. Because $R_{\mathrm{c}}$ is constant, hence $R_{\mathrm{d}}\left(R_{\mathrm{d}}\right.$ $=R-R_{\mathrm{c}}$ ) has similar frequency dependence as $V_{\mathrm{oc}}$ and $R$. The reason for the frequency dependency of $V_{\mathrm{oc}}, R_{\mathrm{d}}$ should be attributed to eddy currents either in the stabilizer layers in the CC or in the iron 'tooth' of the stator. This is a different behaviour with the previously reported flux pumps which used non-stabilized CC stators, where $V_{\mathrm{oc}}$ and $R_{\mathrm{d}}$ were proportional to frequency $[4,6,7]$. The eddy current in the 'tooth' could be eliminated by the use of laminated yokes. However eddy currents in the stabilized layers in CCs cannot be eliminated. Further systematic study in the effect of eddy current will be need to better understand $V_{\mathrm{oc}}$ and $R_{\mathrm{d}}$ behaviours in flux pumps with a magnetic circuit using stabilized CCs. The contact resistance $R_{\mathrm{c}}$ in the circuit can approximately be obtained by the intercept of the linear line fit of $R$ for $g=10.9 \mathrm{~mm}$ on $R$ axis, as influence of eddy current is small at this large $g$. The obtained $R_{\mathrm{c}}$ is $4.2 \mu \Omega$ for the flux pump. This $R_{\mathrm{c}}$ value is substantially larger than values used in previous works, and is a result of non-optimal soldering on the experimental circuit reported here $[3,4,6,7]$. Despite this 

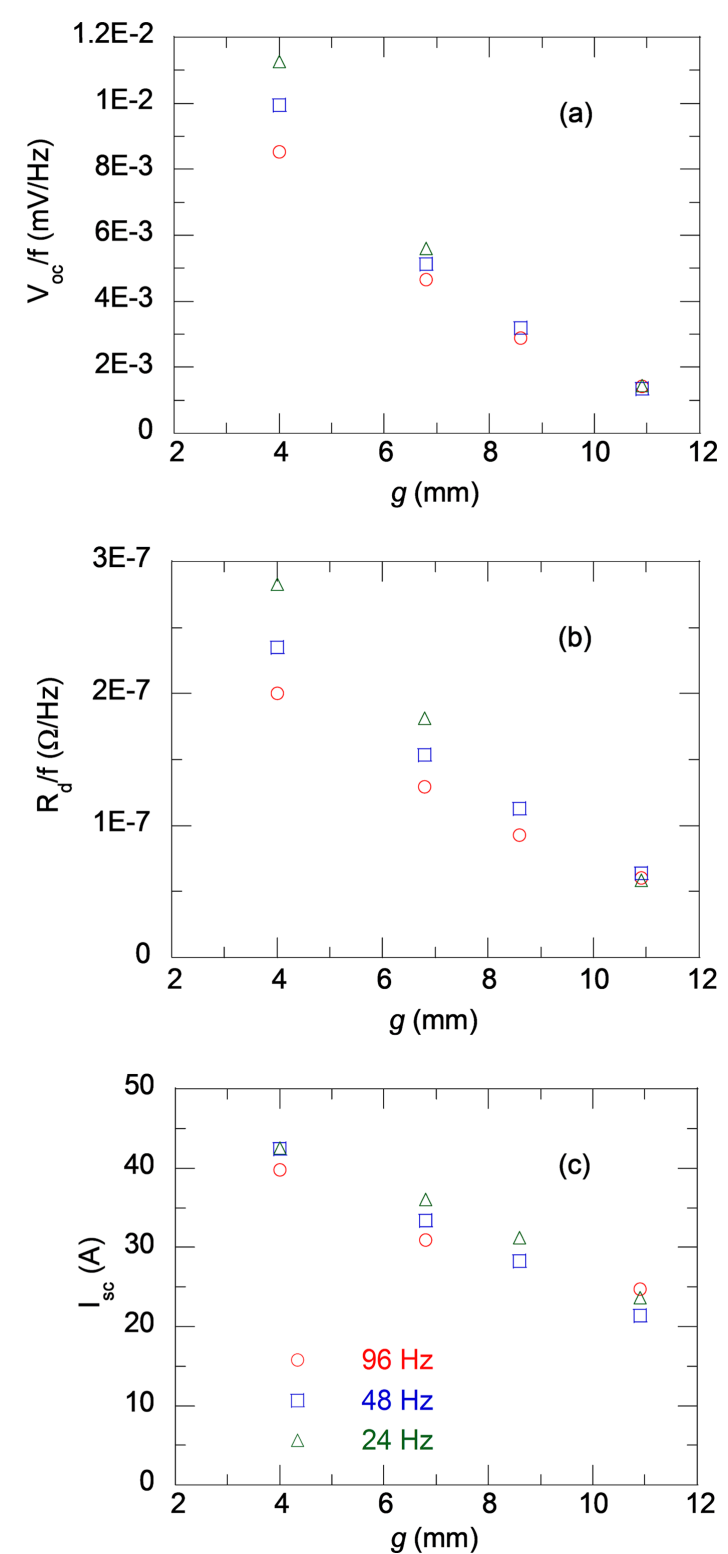

Fig. 4. (Color online) (a) $V_{\text {oc }} / f$ vs $g$ at various frequencies; (b) $R_{\mathrm{d}} / f$ vs $g$ at various frequencies; (c) $I_{\mathrm{sc}}$ vs $g$ at various frequencies.

large series resistance we see that the flux pump remains capable of injecting current into the HTS coil across the entire range of operating parameters surveyed here.

Figure 4 shows plots of the normalized values of $V_{\mathrm{oc}}, R_{\mathrm{d}}$ by $f$, and $I_{\mathrm{sc}}\left(I_{\mathrm{sc}}=V_{\mathrm{oc}} / R_{\mathrm{d}}\right)$, which have been extracted from Fig. 2(c) considering the $R_{\mathrm{c}}$ value. The data are plotted as a function of $g$. There is difference between $V_{\text {oc }} / f$ values for different frequencies as well as between $R_{\mathrm{d}} / f$ values for a given flux gap. The value is smallest when the frequency is the largest. The difference becomes small with increasing $g$. At $g=10.9 \mathrm{~mm}$, the difference dis- appears almost completely. This is consistent with the results shown in Fig. 3 which we attributed to eddy currents. Even though there is notable eddy current effect on $V_{\mathrm{oc}}$ and $R_{\mathrm{d}}$, we observe that $I_{\mathrm{sc}}$ is approximately independent of frequency all flux gaps (see Fig. 4(c)). This results from the fact that $I_{\mathrm{sc}}$ is determined by $V_{\mathrm{oc}} / R_{\mathrm{d}}$. It has been shown that both $V_{\mathrm{oc}}$ and $R_{\mathrm{d}}$ are proportional to the applied field amplitude, $B$. in the presence of eddy currents the applied field is reduced, thus leading to a proportional reduction in both $V_{\mathrm{oc}}$ and $R_{\mathrm{d}}$. However these effects cancel each other out in the ratio $V_{\mathrm{oc}} / R_{\mathrm{d}}$ so that $I_{\mathrm{sc}}$, remains unaffected by the presence of eddy current effects. As $I_{\mathrm{sc}}$ represents the maximum output current available from the flux pump, this result implies that eddy current effects do not impact on the current pumping capacity of the flux pump, and hence both ferromagnetic materials and stabilized wires can be used for HTS flux pump applications.

The $I_{\text {sc }}$ values shown in Fig. 4(c) decrease with increasing the flux gap, which means $V_{\text {oc }}$ decreases more rapidly with increasing $g$ than is the case for $R_{\mathrm{d}}$. The $I_{\mathrm{sc}}$ values at $10.9 \mathrm{~mm}$ are considerably large compared to that in the barrel flux pump without a magnetic circuit where $I_{\mathrm{sc}}$ was less than $10 \mathrm{~A}$ at $g=7.5 \mathrm{~mm}[6]$.

\section{Conclusion}

In this work, we have demonstrated a mechanically rotating flux pump employing a ferromagnetic magnetic circuit and a Cu-stabilized coated conductor (CC) HTS stator. We have shown that the flux pump can be described by the same single circuit model which we used to describe barrel-type flux pumps, where flux pumps are composed of a DC open-circuit and an internal resistance due to dynamic resistance. The DC open-circuit voltage and internal resistance exhibit a non-linear dependence with operating frequency, which is due to eddy currents either in the stator yoke of the flux pump or in the stabilizing layers of the coated conductor. However, and importantly, the eddy currents have little effect on the maximum output current available from the flux pump and hence both ferromagnetic materials and stabilized coated conductors could be used for HTS flux pump application. The flux pump described here shows superior current injecting capability over barrel-type flux pumps without a magnetic circuit which have been reported previously, and is a promising architecture for the development of mechanically rotating flux pumps which can operate at large flux gaps, such that the mechanically rotating parts can be located outside of the cryogenic enclosure. 


\section{References}

[1] L. J. M. van de Klundert, and H. H. J. ten Kate, Cryogenics 21, 195 (1981).

[2] S. Lee, W. S. Kim, Y. Kim, J. Y. Lee, S. H. Park, J. K. Lee, G. W. Hong, S. S. Kim, Y. J. Hwang, and K. Choi, IEEE Trans. Appl. Supercond. 26, 0606104 (2016).

[3] C. Hoffmann, D. Pooke, and A. D. Caplin, IEEE Trans. Appl. Supercond. 21, 1638 (2011).

[4] Z. Jiang, K. Hamilton, N. Amemiya, R. A. Badcock, and C. W. Bumby, Appl. Phys. Lett. 105, 112601 (2014).

[5] T. A. Coombs, J. F. Fagnard, and K. Matsuda, IEEE
Trans. Appl. Supercond. 24, 8201005 (2014).

[6] Z. Jiang, C. W. Bumby, R. A. Badcock, H. J. Sung, N. J. Long, and N. Amemiya, Supercond. Sci. Technol. 28, 115008 (2015).

[7] Z. Jiang, C. W. Bumby, R. A. Badcock, and H. J. Sung, IEEE Trans. Appl. Supercond. 26, 4900706 (2016).

[8] C. W. Bumby, Z. Jiang, J. E. Storey, A. E. Pantoja, and R. A. Badcock, Appl. Phys. Lett. 108, 122601 (2016).

[9] K. Ogasawara, K. Yasukochi, S. Nose, and H. Sekizawa, Cryogenics 16, 33 (1976).

[10] M. P. Oomen, J. Rieger, M. Leghissa, B. ten Haken, and H. H. J. ten Kate, Supercond. Sci. Technol. 12, 382 (1999). 